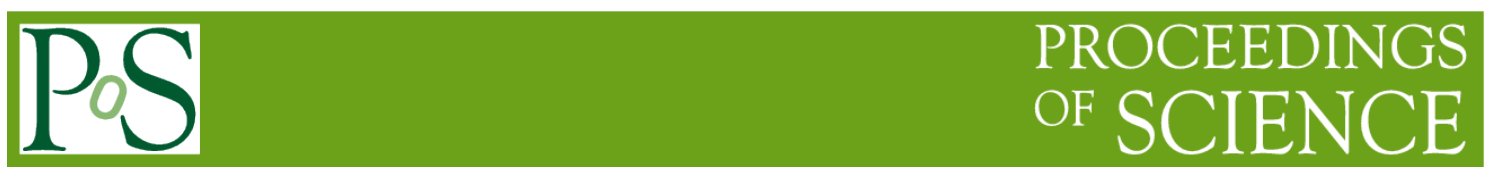

\title{
Radio Astronomy and Innovation
}

\section{Arnold van Ardenne}

ASTRON

Oude Hoogeveensedijk 4, 7991 PD Dwingeloo, The Netherlands

E-mail: ardenne@astron.nl

\section{Carole Jackson}

ASTRON

Oude Hoogeveensedijk 4, 7991 PD Dwingeloo, The Netherlands

E-mail: jackson@astron.nl

\section{Dion Kant}

Formerly ASTRON Eng. Project Leader

E-mail: g.w.kant@hunenet.nl

\section{Parbhu Patel}

Formerly ASTRON Eng. Project Leader

E-mail:parbhup@sky.com

50 Years Westerbork Radio Observatory, A Continuing Journey to Discoveries and Innovations Richard Strom, Arnold van Ardenne, Steve Torchinsky (eds) 


\title{
Chapter 16 Radio Astronomy and Innovation
}

\author{
Arnold van Ardenne, Carole Jackson*
}
T $\mathrm{t}$ can be asked, "What is it good for and why invest in radio astronomy?" Is it sufficient that it is the singular pursuit of knowledge? Or does it have more value, and if so, how do we assess its impact on our lives and the wider society?

There are some simplistic and perhaps satisfactory answers to the first part of the question: radio astronomy is a curiosity-led science and salves our collective want to know more about our Universe. However, the question is more usually asked from a different perspective and this simplistic answer does not always suffice: We need to be far more precise. For example, we know that the enabling factor for the good use of public funds is underpinned by governmental enquiries. These enquiries are driven in such a way as to yield valuable answers. At the same time, the political context can change, such that we need to be fully prepared to promote this investment to the general public, who after all are the ultimate funders as taxpayers.

\section{Investing for new knowledge}

A hundred years ago, mankind was unaware of the scale of the Universe, its constituent billions of galaxies and only had a general belief that the cosmos was 'very old'. Today we have a confident measure of its age and its likely evolutionary path. We observe the physical manifestations of its constituent objects (stars, galaxies, clusters, etc) across the entire electromagnetic (EM) spectrum, from radio to hard x-rays, as well as use non-EM instruments to detect particles, cosmic rays and gravitational wave ripples of ongoing and cataclysmic events. It is no exaggeration to say that our understanding of the cosmos has made huge strides through the last two decades. Massive surveys have been compiled using new detector technologies, advancing our knowledge of the gross structure and evolution of the Universe. Alongside this, non-EM detectors have allowed the detection of gravitational waves confirming Einstein's fundamental predictions of 100 years prior (2016), earth-like planetary systems have been located and dark energy is both inferred and generally accepted. As a result, we live in an era where astronomy continues to mature at a rapid pace, probing ever more extreme tests of fundamental physics. Radio astronomy is a relative-

* ASTRON, The Netherlands ly young aspect of astronomy, having developed rapidly over the last 80 years or so. It is both remarkable, and a note to the unique discovery space of the radio 
regime, that $80 \%$ of the Nobel prizes awarded to astronomical subjects are in the radio regime of astronomy. This is partly supported because of its huge, five orders of frequency spectral coverage observable by using ground-based instrumentation.

To arrive and remain at the front line of observational discoveries, this groundbased instrumentation must be leading edge and, by implication, innovative. For example, the WSRT was precisely on time because for 10 years, it was the most sensitive synthesis radio telescope in the world enabling spectacular discoveries to be made. It continued to push frontiers induced by a climate of close collaboration between scientist and engineers, many of whom also taught at universities and educational institutes and had close connections to industry.

I once challenged Ger de Bruyn with the "why invest" question. His reply was more or less a solid "because pure science deserves to be funded as it appeals on mankind's universal curiosity and mankind wants to understand nature". True as that may be and as important as it is, more is required to allow this statement to bear fruits. For example, not only is there a significant financial commitment necessary to enable excellent radio astronomy in the Netherlands, it relies on the educational excellence at the Dutch universities. These work as a

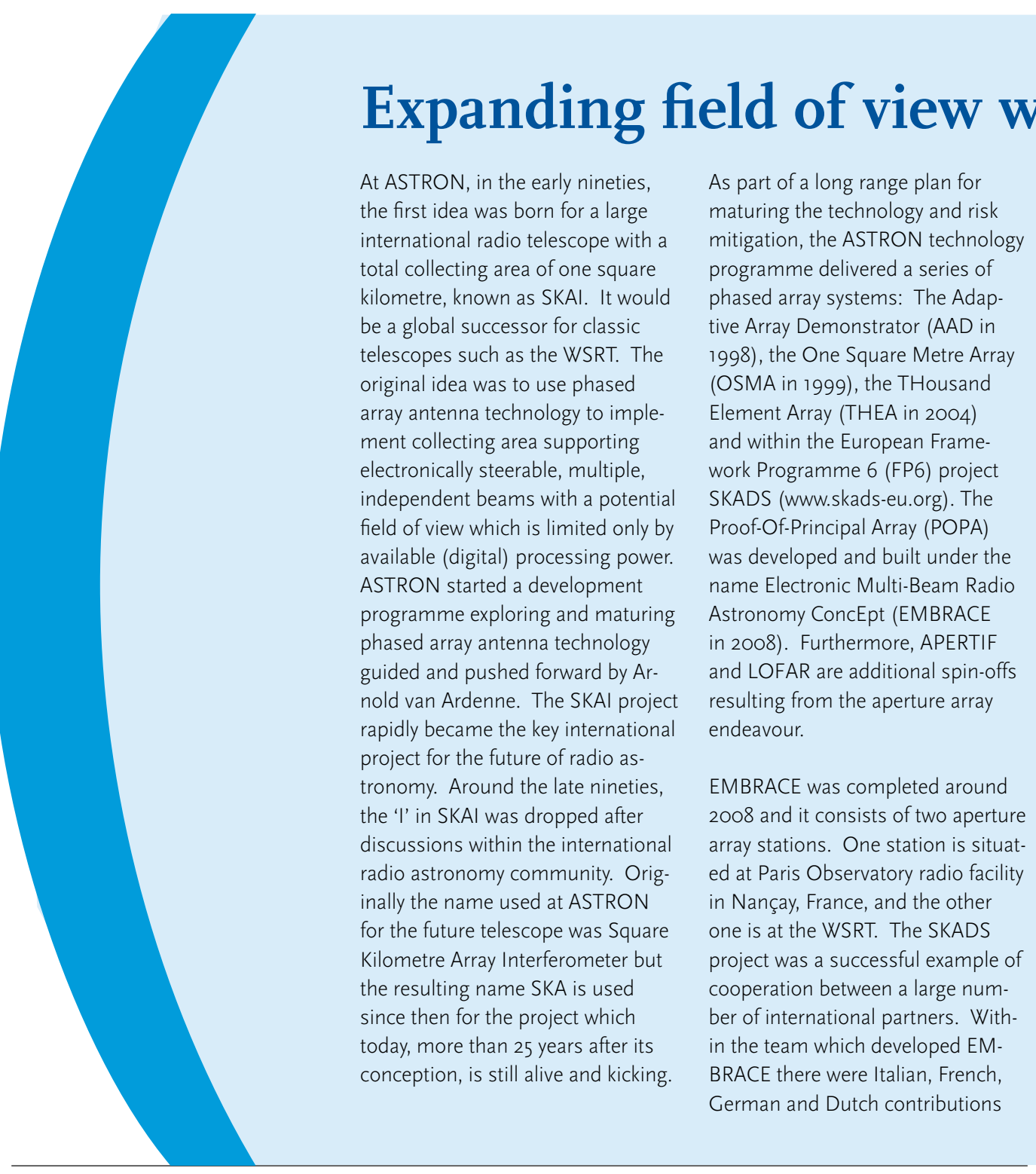

Dion Kant, Parbhu Patel (both formerly at ASTRON)

block of the antenna array. It operates in the frequency range of 500 to $1500 \mathrm{MHz}$ using tapered slot antenna elements known as Vivald elements.

The station in the Netherlands, shown in Fig. 1, is situated between telescope 4 and 5 of the WSRT. It has an aperture of about $160 \mathrm{~m}^{2}$, an effective noise system temperature less than $100 \mathrm{~K}$ and it provides two independent analogue beams. Each beam has a field of view (FoV) of more than 200 square degrees and this is a result of the size of the tiles used as the basic building

A novel radome concept was used where polystyrene plates were pushed into a curved global cover over the antenna system to protect it from the weather conditions. However, during the first winter, heavy snowfall damaged the radome. After fixing the damaged radome, it has survived all subsequent winters to-date. The radome for the station in Nançay was built slightly after the one built at the WSRT. The quality of the polystyrene material used for the radome in Nançay was much better and after about ten years the station in Nançay is still in optimal shape. The EMBRACE station in Nançay continued until very recently with various astronomical observations (see Torchinsky et al, 2016, A\&A, 589 , A77) as the largest operating AA-station in the world!

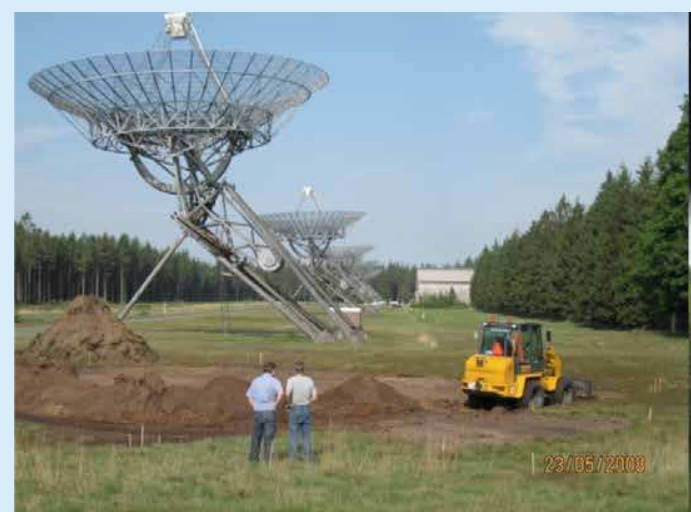

Turning the soil at the location between WSRT Telescopes 4 and 5 for the first EMBRACE station, while Jan Pister
de Reijer and Dion Kant are supervising the process. An impression of the EMBRACE station configuration showin the radome and the cabin with the processing system on the right. Two beams are depicted showing the capability of one beam being used for $\mathrm{Hl}$ imaging while the other beam is being used for observing pulsars. 
positive feedback system. In this manner, top-level astronomy research and education systems produce top students who develop to become top researchers and so on. Furthermore, the international outlook helps in acquiring European funds to do further research, here or elsewhere with a wide range of collaborators. Dutch radio astronomy has long supported the route to move overseas after a PhD, to connect and learn, and possibly to return later.

\section{Collaboration and society}

We can provide a heuristic definition of Radio Astronomy as the manipulation of increasingly larger datasets from observations of the Universe in the radio regime of the electromagnetic spectrum to provide meaningful and scientifically justifiable data, interpretations and discoveries. For example, working at the edge of the capabilities of new observing instruments such as LOFAR and in future the SKA, these processes involve intense data mapping onto the highest performance computing platforms.

Therefore, whilst radio astronomy is a curiosity driven science, it fundamentally demands technologies and directed advances in radio instrumentation, signal-processing and high performance computing and imaging techniques to achieve its discoveries. Exploring this idea a bit further, a wide range of technological challenges, entrepreneurship and shared talents are the basis for collaborations besides funding, providing fertile ground from which impactful discoveries eventuate. This defines the rich innovative domain with wider applications illustrated below.

Radio and therefore, radio techniques are the primary sensors underlying radio astronomical discoveries. Both are elements of our present-day telecommunication infrastructure, but at the birth of radio astronomy in the 1940's they were not well developed. As a result, many aspects of our telecom systems almost immediately profited from radio astronomical instrumental developments: These range from fully steerable antennas, low noise receivers and antennas. It goes beyond this chapter to follow these lines in any detail so a most recent example may illustrate this.

In this book, the chapter on Apertif describes a new observing system for the WSRT. Apertif adopts a multi-pixel radio "camera" receiver as an upgrade to the single pixel (horn) receivers traditionally used in this part of radio frequency domain. Early technical R\&D towards this multi-pixel system started at ASTRON as part of first phase SKA R\&D in the late 1990's and has now matured to the point of being implemented as a new radio astronomical instrument. It is exactly this same principle of the focal plane arrays that now has found a suggested application in the next generation (" $5 G$ ") network infrastructure through work at the technical University Eindhoven.

Thanks to its ultra-wide viewing angle on the sky, a similar technique without the use of a reflector antenna is termed an aperture array. This has been imple- mented as LOFAR and other more recent low frequency telescopes worldwide This technique is now proposed for simultaneous multi satellite observations in ground station applications and was first demonstrated at ASTRON around 2001.

Besides the use in the "physical layer" of the telecom infrastructure as illustrat ed above, other applications originate from the use of optimized algorithms and imaging techniques now also used in MRI apparatus. Research starting in ASTRON in the early eighties by John O'Sullivan, now in Australia, ultimately contributed to the innovation of wireless computing (WiFi) many years later. Moving further toward the computing infrastructure and intense data processing, collaborations with IBM into the next, exa-scale computing platform and some enabling technologies (see: http://www.dome-exascale.nl/) naturally connect future radio astronomy needs with generic computing requirements e.g. required for broader, demanding applications beneficial to society.

For the last two decades ASTRON has maintained a structured effort to strengthen the relations both to engineering societies and institutes for technology and education as well as to the users i.e. companies and industrial domains. First, by establishing a technology transfer platform and later a private company (AstroTech) through which now LOFAR stations are sold. The latter is a great success and most is acquired from industry; a true "export product". Nationally, by virtue of the breadth of science enabled by ASTRON facilities and the technological innovation required to develop them, ASTRON is deeply embedded in the National Science Agenda (NWA) as well as to the Universities. Also, ASTRON collaborates with the astroparticle physics community, via NWO institute as mother organization, NIKHEF and others, to further research on gravitational waves and cosmic rays, and with KNMI and NLR for research on Space Weather.

Finally, ASTRON collaborates with the four NL Technical Universities and the NL eScience Centre on science areas relevant to innovation towards the future generation of radio facilities including radio science, antennas, photonics, signal processing technologies, distributed algorithms and high-performance computing all with relevant associated application domains and often in collaboration with industry.

\section{Application domains for Innovation}

Capturing the specific and broader, societal impacts of radio astronomy is not trivial. An attempt is shown in the picture below which is inspired by an insightful presentation some years ago by Simon Garrington now Associate Director for Jodrell Bank Observatory in the UK. 


\begin{tabular}{|c|c|c|}
\hline Radio Astronomy & Technological Spinoff & Application \\
\hline $\begin{array}{l}\text { Radio Interferometry } \\
\text { Low Frequency } \\
\text { Interferometry (LOFAR) }\end{array}$ & $\begin{array}{l}\text { Wireless LAN technology } \\
\text { Location of wireless sensor nodes } \\
\text { Sun-Earth Interaction, Ionosphere }\end{array}$ & $\begin{array}{l}\text { WiFi Internet networking } \\
\text { e.g. Location of mobile emergency calls } \\
\text { Critical infrastructures, Safety\&Security }\end{array}$ \\
\hline $\begin{array}{l}\text { Very Long Baseline } \\
\text { Interferometry } \\
\text { Precision astrometry }\end{array}$ & $\begin{array}{l}\text { Most accurate (hydrogen maser) clocks } \\
\text { International celestial reference frame. GPS } \\
\text { reference frames } \\
\text { Precision State Vector/position }\end{array}$ & $\begin{array}{l}\text { Space communication, satellite navigation } \\
\text { Earth orientation parameters, geodesy, time } \\
\text { keeping } \\
\text { Interplanetary spacecraft navigation, Planetary } \\
\text { science }\end{array}$ \\
\hline $\begin{array}{l}\text { Aperture synthesis and } \\
\text { image reconstruction }\end{array}$ & $\begin{array}{l}\text { (Fourier) imaging techniques } \\
\text { Massive Data, Algorithms \& Database } \\
\text { Thinned aperture imaging radiometry }\end{array}$ & $\begin{array}{l}\text { Medical imaging tomography } \\
\text { Image de-blurring } \\
\text { (Green) Computing, Wireless Computing } \\
\text { Earth sciences, Oceanography (HI-freq. band) }\end{array}$ \\
\hline $\begin{array}{l}\text { (Homologous) antenna } \\
\text { design }\end{array}$ & $\begin{array}{l}\text { Precision antennas } \\
\text { Widefield Antennas } \\
\text { Focal Plane Arrays }\end{array}$ & $\begin{array}{l}\text { Communications and radar } \\
\text { Earth Obs., GNSS (Safety\&Rescue) } \\
\text { 5G(?) }\end{array}$ \\
\hline Low Noise Amplifiers & $\begin{array}{l}\text { Highly sensitive commercial cryogenically } \\
\text { cooled receiving systems }\end{array}$ & $\frac{\text { GALAXYZOO }}{\text {. }}$ \\
\hline Endeavour & Societal, Public & $\begin{array}{l}\text { Education, Inspiration } \\
\text { UNAWE, Astrotechnology }\end{array}$ \\
\hline
\end{tabular}

This again, indicates the broad interest in making explicit connections between science and society and illustrates interconnectedness between astronomical research and technology development.

Our discussion and evaluation of our 'value' becomes ever more relevant as the funding horizon for technology tends to move in closer with less free space to allow the potential for serendipitous $R \& D$ (as the $5 \mathrm{G}$ example and the wireless application example showed with lead times of 15-20 years). Effectivity therefore is hard to measure on a short term timescale, contrasting perhaps results and efficiency.

Whilst an assessment of the scientific (pure and technological) endeavour of radio astronomy's long-term impact is best done in retrospect as is illustrated in the earlier picture, it is clear that for innovation to work, a mix and match of research and technology is essential. 